\title{
SUPPLEMENTARY METERIAL
}

\section{A novel pyrrole alkaloid from the fruit peels of Strychnos nux-blanda}

Jirapast Sichaem $^{\mathrm{a}}$, Kornkanok Ingkaninanand ${ }^{\mathrm{b}}$ and Santi Tip-pyang ${ }^{\mathrm{a},{ }^{*}}$

${ }^{a}$ Natural Products Research Unit, Department of Chemistry, Faculty of Science,

Chulalongkorn University, Bangkok 10330, Thailand

${ }^{b}$ Department of Pharmaceutical Chemistry and Pharmacognosy, Faculty of Pharmaceutical Sciences, Naresuan University, Phitsanulok 65000, Thailand

\section{Corresponding author}

Assoc. Prof. Dr. Santi Tip-pyang, Natural Products Research Unit, Department of Chemistry, Faculty of Science, Chulalongkorn University, Bangkok 10330, Thailand, Tel.: +662 218 7625; fax +662 218 7598; E-mail address: $\underline{\text { Santi.Ti@chula.ac.th }}$

\begin{abstract}
A novel pyrrole alkaloid, strychnuxin (1), along with five known compounds (2-6) were isolated from the fruit peels of Strychnos nux-blanda. The structures of all the isolated compounds (1-6) were fully characterized using spectroscopic data, as well as comparisons with the previous literature data. Moreover, all isolated compounds were assessed for their $\alpha$ glucosidase and acetylcholinesterase inhibitory activities.
\end{abstract}

Keywords: Strychnos nux-blanda, Loganiaceae, Pyrrole alkaloid, Anti- $\alpha$-Glucosidase activity, Antiacetylcholinesterase activity. 


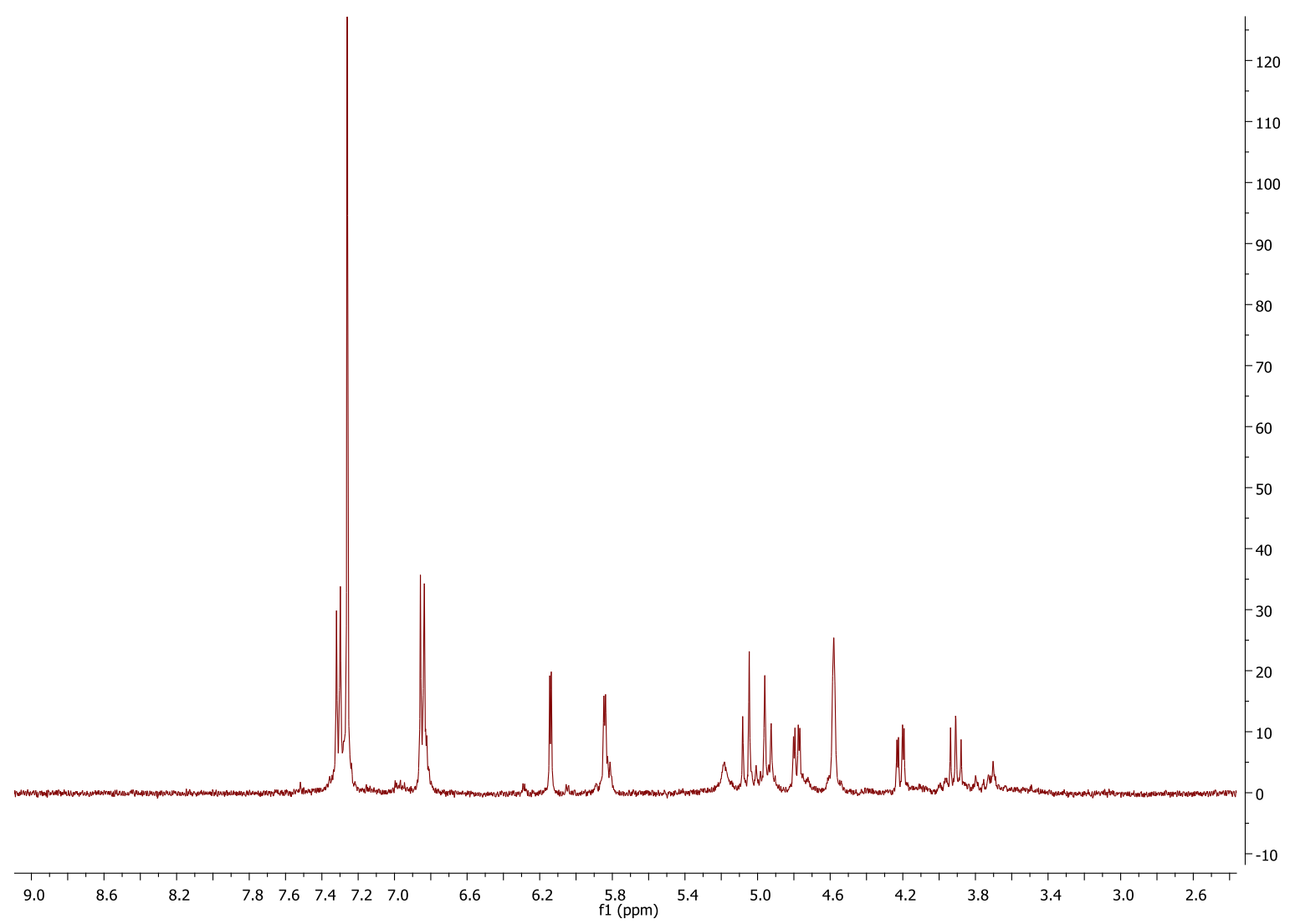

Figure S1. ${ }^{1} \mathrm{H}$ NMR spectrum of $\mathbf{1}$ in $\mathrm{CDCl}_{3}$.

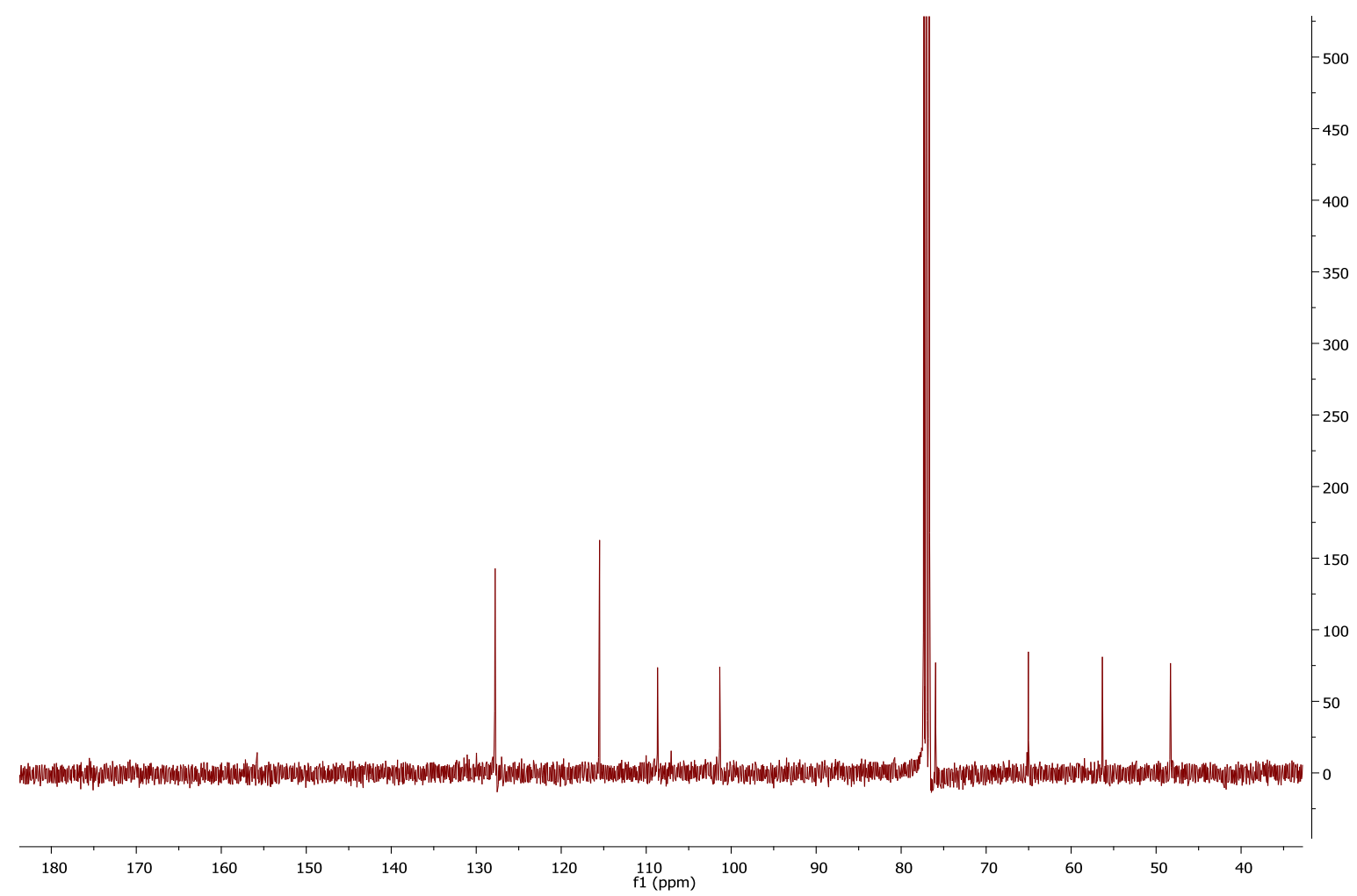

Figure S2. ${ }^{13} \mathrm{C}$ NMR spectrum of $\mathbf{1}$ in $\mathrm{CDCl}_{3}$. 


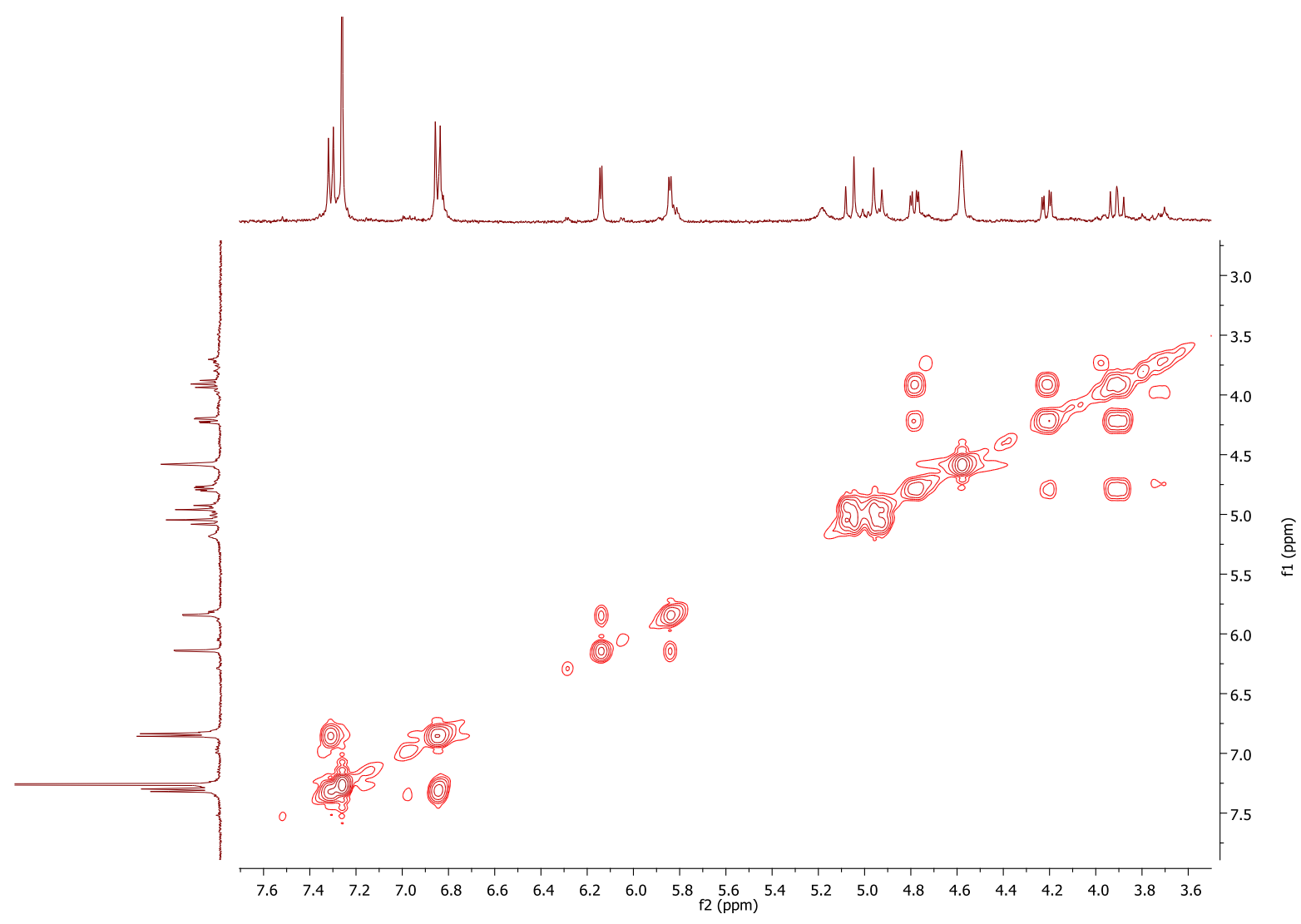

Figure S3. COSY NMR spectrum of 1 in $\mathrm{CDCl}_{3}$.

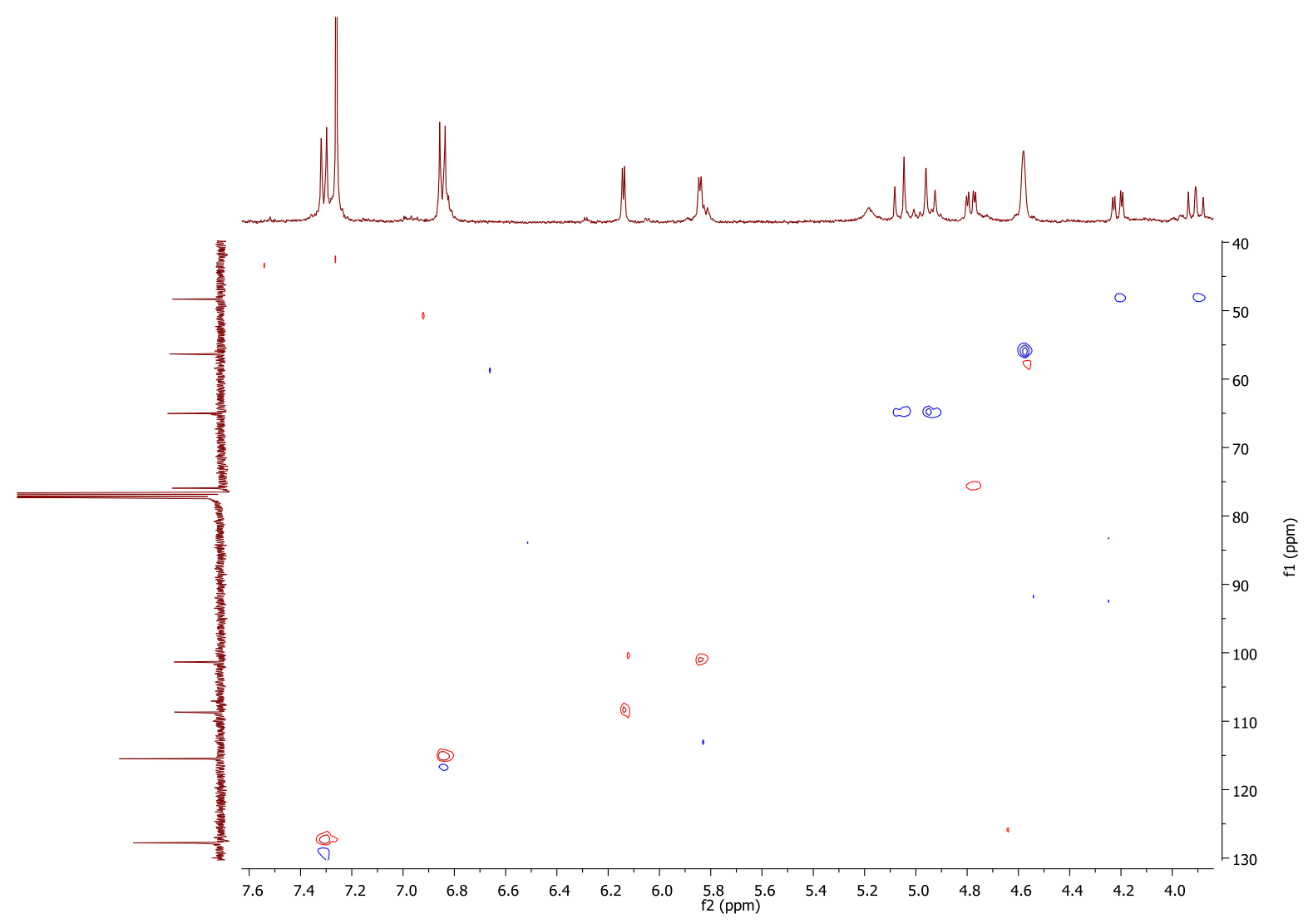

Figure S4. HSQC NMR spectrum of 1 in $\mathrm{CDCl}_{3}$. 


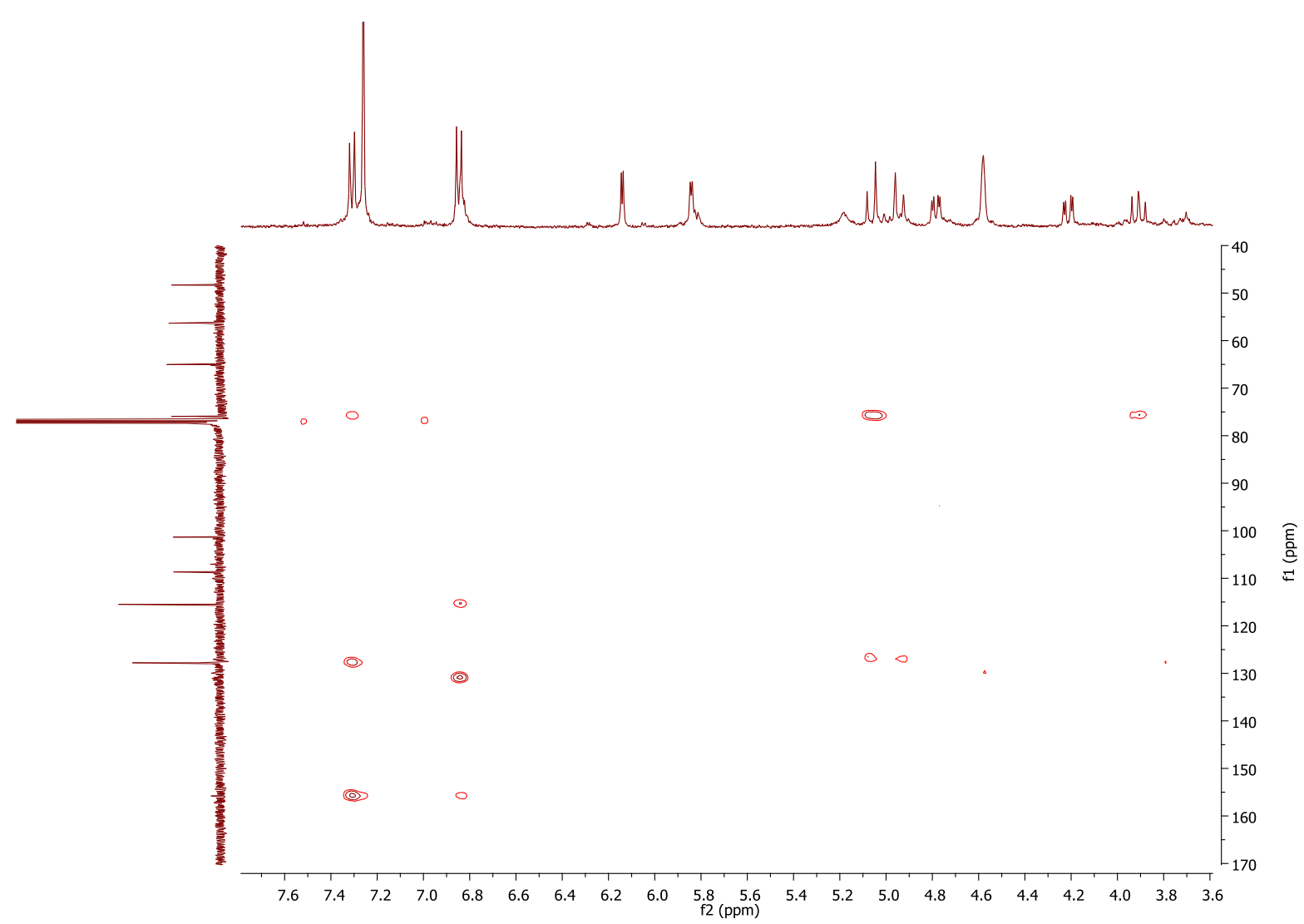

Figure S5. HMBC NMR spectrum of 2 in $\mathrm{CDCl}_{3}$.

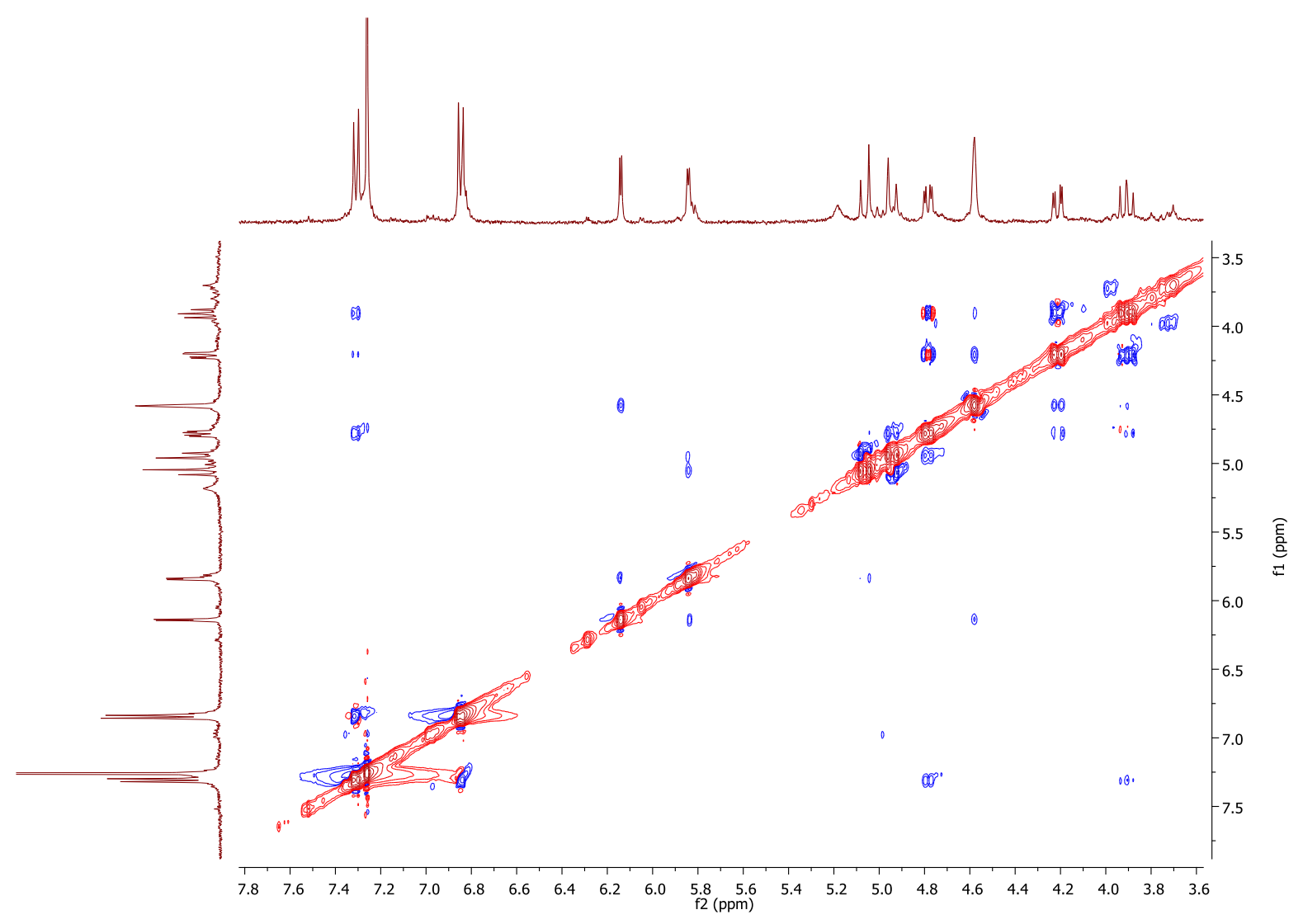

Figure S6. NOESY NMR spectrum of $\mathbf{1}$ in $\mathrm{CDCl}_{3}$ 

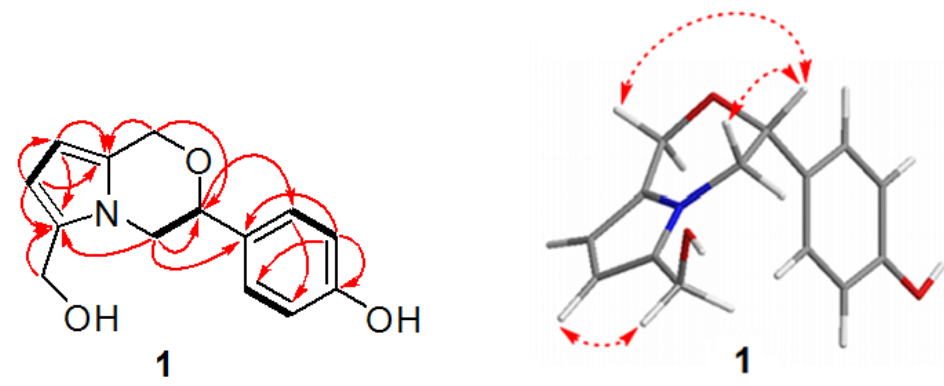

Figure S7. Selected HMBC (arrows), COSY (bold lines) and NOESY correlations of 1.

Table S1. ${ }^{1} \mathrm{H}$ and ${ }^{13} \mathrm{C}$ NMR data of $\mathbf{1}$ in $\mathrm{CDCl}_{3}\left(400 \mathrm{MHz}\right.$ for ${ }^{1} \mathrm{H}, 100 \mathrm{MHz}$ for ${ }^{13} \mathrm{C}$ ).

\begin{tabular}{|c|c|c|}
\hline \multirow{2}{*}{ Position } & \multicolumn{2}{|l|}{1} \\
\hline & $\overline{\delta_{\mathrm{H}}(\mathrm{ppm}), J(\mathrm{~Hz})}$ & $\delta_{\mathrm{C}}(\mathbf{p p m})$ \\
\hline 2 & - & 131.0 \\
\hline 3 & $6.14, \mathrm{~d}(3.4)$ & 108.7 \\
\hline 4 & $5.84, \mathrm{~d}(3.4)$ & 101.3 \\
\hline 5 & - & 131.4 \\
\hline $6 a$ & $5.06, \mathrm{~d}(14.4)$ & 65.0 \\
\hline $6 b$ & $4.94, \mathrm{~d}(14.4)$ & \\
\hline 8 & $4.78, \mathrm{dd}(11.0,3.4)$ & 75.9 \\
\hline $9 \mathrm{a}$ & $4.21, \mathrm{dd}(12.2,3.4)$ & 48.3 \\
\hline $9 b$ & $3.91, \mathrm{dd}(12.2,11.0)$ & \\
\hline $1^{\prime}$ & - & 129.9 \\
\hline $2^{\prime}, 6^{\prime}$ & $7.31, \mathrm{~d}(8.4)$ & 127.8 \\
\hline $3^{\prime}, 5^{\prime}$ & $6.86, \mathrm{~d}(8.4)$ & 115.5 \\
\hline $4^{\prime}$ & - & 155.7 \\
\hline $2-\mathrm{CH}_{2} \mathrm{OH}$ & $4.57 \mathrm{~d}(4.0)$ & 56.3 \\
\hline
\end{tabular}

